\title{
Die Kristall- und Molekülstruktur von Bis(trimethylsilyl)diimin
}

\author{
Von M. VeITH UNd H. BäRNighauSEN \\ Institut für Anorganische Chemie der Universität Karlsruhe $(T H)$, \\ D75 Karlsruhe, Englerstrasse 11, Deutschland (BRD)
}

(Eingegangen am 8. Februar 1974; angenommen am 7. März 1974)

\begin{abstract}
The crystal structure of bis(trimethylsilyl)diimine, $\left(\mathrm{CH}_{3}\right)_{3} \mathrm{Si}-\mathrm{N}=\mathrm{N}-\mathrm{Si}\left(\mathrm{CH}_{3}\right)_{3}$, has been determined from three-dimensional X-ray data collected on a Stoe two-circle diffractometer at $-130^{\circ} \mathrm{C}$ (Mo $K \alpha$ radiation, 673 reflexions, $R=0.067$ ). The crystals are monoclinic, space group $P 2_{1} / c$, with cell dimensions $a=6.12(2), b=10.788(3), c=8.892$ (3) $\AA$, and $\beta=103.4(1)^{\circ}$. There are only two molecules in the unit cell and, consequently, the crystal site symmetry must be $\bar{T}\left(C_{i}\right)$. For several reasons, however, the free molecule is to be expected to adopt the higher point symmetry $2 / m\left(C_{2 h}\right)$. Important molecular dimensions are the very short $\mathrm{N}-\mathrm{N}$ bond $(1 \cdot 17 \AA)$, the unusual long $\mathrm{Si}-\mathrm{N}$ bond $(1.81 \AA)$, and the $\mathrm{Si}-\mathrm{N}-\mathrm{N}$ angle of $120^{\circ}$, which indicate the exceptional position of this compound among the other known trans $-\mathrm{X}-\mathrm{N}=\mathrm{N}-\mathrm{X}$ systems. It is shown that some geometrical details of the crystal and molecular structure result from the stereochemical activity of the nitrogen lone pair.
\end{abstract}

\section{Einleitung}

Obwohl es in der Vergangenheit nicht an Versuchen gefehlt hatte, in Analogie zu den lange bekannten Azoalkanen $\mathrm{R}_{3} \mathrm{C}-\mathrm{N}=\mathrm{N}-\mathrm{CR}_{3}$ die entsprechenden Azosilane $\mathrm{R}_{3} \mathrm{Si}-\mathrm{N}=\mathrm{N}-\mathrm{Si} \mathrm{R}_{3}$ zu synthetisieren, konnte erst vor wenigen Jahren mit der Darstellung von Bis.(trimethylsilyl)diimin der Existenznachweis für einen Vertreter dieser Verbindungsklasse erbracht werden (Wiberg, Joo \& Uhlenbrock, 1968).

Bis(trimethylsilyl)diimin $\quad\left(\mathrm{CH}_{3}\right)_{3} \mathrm{Si}-\mathrm{N}=\mathrm{N}-\mathrm{Si}\left(\mathrm{CH}_{3}\right)_{3}$ - eine hellblaue, bei $-3^{\circ} \mathrm{C}$ schmelzende, leicht zersetzliche und ausserordentlich reaktionsfähige Verbindung - wurde in der Folgezeit von Wiberg und seinen Mitarbeitern intensiv untersucht. Die Bemühungen konzentrierten sich dabei auf das Studium der vielfältigen Reaktionsmöglichkeiten der neuen Verbindung, jedoch wurden auch verschiedene spektroskopische Methoden herangezogen, um Aufschlüsse über die Molekülstruktur von Bis(trimethylsilyl)diimin $\mathrm{zu}$ erhalten. Leider konnte das Strukturproblem auf diese Weise nicht befriedigend gelöst werden. So musste Wiberg (1971) in seinem zusammenfassenden Artikel über Bis(trimethylsilyl)diimin noch offenhalten, ob sich die Trimethylsilylgruppen in cis-oder transStellung zueinander befinden. Unsicher blieb auch die Aussage über den Winkel $\mathrm{Si}-\mathrm{N}-\mathrm{N}$, für den Wiberg (1971) in Anlehnung an die geometrischen Verhältnisse bei Trimethylsilyl-isocyanat und -isothiocyanat (Kimura, Katada \& Bauer, 1966) etwa $150^{\circ}$ annahm.

Trotz der zu erwartenden experimentellen Schwierigkeiten haben wir daher eine röntgenographische Kristallstrukturanalyse in Angriff genommen, um auf diesem Wege zur Klärung des Problems der Molekülstruktur von Bis(trimethylsilyl)diimin beitragen zu können.

\section{Experimentelles und Kristalldaten}

Bis(trimethylsilyl)diimin wurde nach Wiberg, Joo \& Uhlenbrock (1968) durch Umsetzung von Lithiumtris(trimethylsilyl)hydrazid mit Tosylazid dargestellt. Geeignete Einkristalle der Verbindung erhielten wir aus etwa 0,1 molaren n-Pentan-Lösungen bei langsamer Abkühlung auf $-70^{\circ} \mathrm{C}$. Nachdem wir die Hauptmenge des Lösungsmittels durch Dekantieren entfernt hatten, wurden anhaftende Lösungsmittelreste bei $-55^{\circ} \mathrm{C}$ unter einem möglichst genau eingehaltenen Druck von $0,13 \mathrm{~Pa}$ abgezogen. Im Kölbchen verblieben hellblaue, gut voneinander isolierte, quaderförmige Kristalle von 0,5 bis $1 \mathrm{~mm}$ Kantenlänge, die unter Stickstoff als Schutzgas bei der Temperatur von festem Kohlendioxid ('Trockeneis', $-78^{\circ} \mathrm{C}$ ) längere Zeit aufbewahrt werden konnten.

$\mathrm{Da}$ sich Bis(trimethylsilyl)diimin schon unterhalb seines Schmelzpunkts $\left(-3^{\circ} \mathrm{C}\right)$ von etwa $-35^{\circ} \mathrm{C}$ an langsam zersetzt (Wiberg, 1971), durften die Kristalle bei der Auswahl, Abfüllung in Mark-Röhrchen und bei den röntgenographischen Untersuchungen niemals die genannte Temperatur überschreiten. Zugleich mussten sie sich bei allen Manipulationen unter einer trockenen Schutzgasatmosphäre befinden; denn Bis(trimethylsilyl)diimin reagiert äusserst heftig mit Sauerstoff und hydrolysiert leicht. Mit Hilfe der in Fig. 1 dargestellten, im folgenden näher erläuterten Hilfseinrichtung gelang es uns, die eben genannten Bedingungen einzuhalten, ohne dass dabei die Kristallauswahl und -abfüllung sonderlich erschwert worden wäre.

Die einfache Hilfseinrichtung besteht im wesentlichen aus einem Zuleitungsrohr für kalten Stickstoff $\left(-180^{\circ} \mathrm{C}\right)$, an dessen Kopfstück $A$ ein wannenförmiges Aluminiumblech $B$ angesetzt ist. Ein etwa $3 \mathrm{~cm}$ 
langes, mit Stickstoff gefülltes Mark-Röhrchen $C$ befindet sich mit seinem geschlossenen Ende im Innern des Zuleitungsrohres. Unterhalb der trichterförmigen Erweiterung des Mark-Röhrchens ist ein Ring $D$ aus Plastilina angebracht, durch den das spätere Aufnehmen des Röhrchens mit einer Pinzette und die Befestigung am Goniometerkopf sehr erleichtert wird. Stellt man den durch Verdampfung von flüssigem Stickstoff gewonnenen Gasstrom genügend stark ein, so bleiben die wärme- und luftempfindlichen Kristalle an der Stelle $E$ des Aluminiumblechs längere Zeit unzersetzt liegen. Man kann nun wie üblich die Kristalle durch ein Stereomikroskop betrachten, einen geeigneten Einkristall auswählen und diesen mit einer feinen Metallnadel in das Mark-Röhrchen nach $C$ schieben. Schliesslich hebt man das Mark-Röhrchen am offenen Ende an und schmilzt es mit Hilfe des Gasflämmchens $F$ an der Stelle $G$ ab. Der Teil $C$ des Mark-Röhrchens muss dabei natürlich im Bereich des kalten Stickstoffstroms verbleiben.

Zur Ableitung der Kristalldaten von Bis(trimethylsilyl)diimin fertigten wir mit $\mathrm{Cu} K \alpha$-Strahlung in einer Weissenberg-Kamera mit Kühleinrichtung (Firma Nonius, Delft) bei Drehung des Kristalls um die Stäbchenachse [100] ein Drehkristalldiagramm sowie Weissenberg-Filme der Schichten $0 k l, 1 k l$ und $2 k l$ an. Am Schluss exponierten wir zu Eichzwecken auf eine Zweitaufnahme des Äquators $0 k l$ die Reflexe $h k 0$ eines Quarzkristalls bei Zimmertemperatur (Gitterkonstante $a$ von Quarz: 4,9126 $\AA$ ). Entsprechend der Eichung konnten die Gitterkonstanten $b$ und $c$ sehr viel genauer bestimmt werden als die aus dem Drehkristalldiagramm entnommene Gitterkonstante $a$. Die folgenden Gitterparameter des monoklin kristallisierenden Bis(trimethylsilyl)diimins gelten für die mit der Kühleinrichtung in der Weissenberg-Kamera erzielte Temperatur von $-150^{\circ} \mathrm{C}: a=6,12 \pm 0,02 \AA, b=10,788 \pm 0,003$ $\AA, c=8,892 \pm 0,003 \AA, \beta=103,4 \pm 0,1^{\circ}$.

Eine grobe Abschätzung des Raumbedarfs von Bis(trimethylsilyl)diimin zeigte, dass in der Elementarzelle $\left(V=571,1 \AA^{3}\right)$ nur zwei Formeleinheiten der Verbindung enthalten sein können. Für die berechnete Dichte $D_{x}$ ergibt sich mit $Z=2$ der Wert $1,014 \mathrm{~g} \mathrm{~cm}^{-3}$. Die aus den Weissenberg-Aufnahmen entnommene Flächenstatistik ( $h k l$ alle vorhanden, $h 0 l$ nur mit $l=2 n$ und $0 k 0$ nur mit $k=2 n$ vorhanden) führte eindeutig auf die Raumgruppe $P 2_{1} / c$ (Nr. 14).

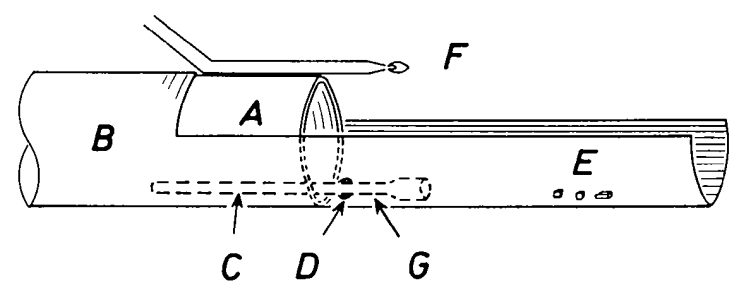

Fig. 1. Kopfstück einer Apparatur, mit deren Hilfe luft- und wärmeempfindliche Kristalle bei etwa $-150^{\circ} \mathrm{C}$ ausgesucht und in Mark-Röhrchen abgefüllt werden können. Nähere Erläuterungen im Text.
Zur Messung der Reflexintensitäten benutzten wir ein automatisches Zweikreis-Diffraktometer der Firma Stoe, Darmstadt (Weissenberg-Prinzip, Mo $K \alpha$-Strahlung, Graphitmonochromator) in Kombination mit der oben erwähnten Kühleinrichtung der Firma Nonius, Delft. Da wir das Diffraktometer ohne einen besonderen Wärmeschutz betrieben, erreichten wir jedoch eine hinreichende Temperaturkonstanz am Kristall nicht wie in der Weissenberg-Kamera bei $-150^{\circ} \mathrm{C}$, sondern erst bei $-130^{\circ} \mathrm{C}$. Der Einkristall hatte die Abmessungen $0,5 \times 0,3 \times 0,3 \mathrm{~mm}$. Die längste Kante entsprach wieder der Richtung [100] und wurde als Drehachse ( $\omega$-Kreis) gewählt. Insgesamt registrierten wir 721 kristallographisch unabhängige Reflexe nach der Technik des ' $\omega$-scans' unter Berücksichtigung der individuellen Reflexbreiten. Der jeweils erforderliche Scan-Bereich wurde nach der Formel $\Delta \omega=1,3+0,7 \cdot \sin v \cos \theta / \sin (Y / 2)$ errechnet; hierbei ist $v$ der Äqui-inklinationswinkel, $\theta$ der Bragg-Winkel, $Y$ der Zählerwinkel, und die Zahlenwerte der Formel sind kristallspezifische, empirisch ermittelte Konstanten in Grad. An den gemessenen Intensitäten wurde die übliche Lorentz- und PolarisationsfaktorKorrektur angebracht. Auf eine Absorptions-Korrektur konnten wir im Hinblick auf die günstige Kristallform und den niedrigen Absorptionskoeffizienten $\mu($ Mo $K \alpha)=2,6 \mathrm{~cm}^{-1}$ verzichten. 29 Reflexe, die sich nicht signifikant vom Untergrund abhoben, wurden als 'nicht beobachtet' eingestuft $\left(F_{o}=0\right) .19 \mathrm{sehr}$ starke Reflexe (in Tabelle 3 mit * gekennzeichnet) hatten zu einer Überlastung des Szintillationszählers geführt und wurden daher bei der Strukturverfeinerung ausgeklammert.

\section{Strukturbestimmung}

Da die Elementarzelle von Bis(trimethylsilyl)diimin 2 Formeleinheiten enthält, die allgemeine Lage der Raumgruppe $P 2_{1} / c$, die für die Atome eines solchen Moleküls nur in Frage kommen kann, aber vierzählig ist, muss ein Symmetrieelement der Raumgruppe zugleich Punktsymmetrieelement des Moleküls sein. Die einzige Symmetrieoperation in $P 2_{1} / c$, die diese Randbedingung erfüllt, ist die Inversion, d.h. Bis(trimethylsilyl)diimin ist ein zentrosymmetrisches Molekül. Als asymmetrische Einheit der Kristallstruktur wählten wir dementsprechend die zu einer Molekülhälfte gehörigen Atome.

Die Ortskoordinaten für das $\mathrm{Si}$-Atom der asymmetrischen Einheit waren aus der Harker-Geraden $P\left(0 v \frac{1}{2}\right)$ und aus der Harker-Ebene $P\left(u \frac{1}{2} w\right)$ einer dreidimensionalen Patterson-Synthese leicht zu entnehmen. Die Positionen des N-Atoms und der drei C-Atome ergaben sich schon ziemlich genau beim Auszeichnen der Minimumfunktion (Buerger, 1967). Sämtliche neun HAtome der asymmetrischen Einheit fanden wir im Anschluss an die Verfeinerung der Orts- und Schwingungstensor-parameter von $\mathrm{Si}, \mathrm{N}, \mathrm{C}(1), \mathrm{C}(2)$ und $\mathrm{C}(3)$ in einer dreidimensionalen Differenz-FourierSynthese. Nach Einbezug der H-Atome in die Ver- 
feinerung unter der Annahme isotroper Temperaturfaktoren für diese Atome verbesserte sich der Gütefaktor $R=\sum|| F_{o}|-| F_{c}|| / \sum\left|F_{o}\right|$ von 0,086 auf den endgültigen Wert 0,067 (die Reflexe mit $F_{o}=0$ wurden bei der Summation ausgeschlossen). Bei den genannten Verfeinerungen nach der Methode der kleinsten Fehlerquadrate minimalisierten wir $\sum\left(|| F_{o} \mid-\right.$ $\left.\left|F_{c}\right| \mid / \sigma\right)^{2}$, wobei wir für die Standardabweichung $\sigma$ der

Tabelle 1. Die Ortskoordinaten und der Parameter des Debye-Waller-Faktors für die Atome der asymmetrischen Einheit von Bis(trimethylsilyl)diimin

Die bei den Atomen $\mathrm{Si}, \mathrm{N}, \mathrm{C}(1), \mathrm{C}(2)$ und $\mathrm{C}(3)$ angegebenen $B$-Werte des Temperaturfaktors der Form exp $\left(-B \sin ^{2} \theta / \lambda^{2}\right)$ wurden nach Hamilton (1959) aus den Werten $\beta_{i, l}$ von Tabelle 2 berechnet. Die Standardabweichungen in Klammern sind entsprechend ihrer Stellenzahl den letzten Ziffern der Funktionswerte zuzuordnen.

\begin{tabular}{llrl} 
& \multicolumn{1}{c}{$\boldsymbol{x}$} & \multicolumn{1}{c}{$y$} & \multicolumn{1}{c}{$z$} \\
$\mathrm{Si}$ & $0,3748(2)$ & $0,1009(1)$ & $0,1725(1)$ \\
$\mathrm{N}$ & $0,4143(6)$ & $0,0241(3)$ & $0,0001(4)$ \\
$\mathrm{C}(1)$ & $0,2165(8)$ & $0,2444(4)$ & $0,1023(5)$ \\
$\mathrm{C}(2)$ & $0,2031(8)$ & $-0,0085(4)$ & $0,2569(5)$ \\
$\mathrm{C}(3)$ & $0,6489(7)$ & $0,1367(4)$ & $0,3052(5)$ \\
$\mathrm{H}(1)$ & $0,085(9)$ & $0,233(5)$ & $0,030(6)$ \\
$\mathrm{H}(2)$ & $0,181(8)$ & $0,285(5)$ & $0,189(6)$ \\
$\mathrm{H}(3)$ & $0,301(9)$ & $0,304(5)$ & $0,047(6)$ \\
$\mathrm{H}(4)$ & $0,176(8)$ & $0,031(5)$ & $0,362(7)$ \\
$\mathrm{H}(5)$ & $0,064(9)$ & $-0,021(4)$ & $0,184(6)$ \\
$\mathrm{H}(6)$ & $0,280(9)$ & $-0,075(5)$ & $0,267(5)$ \\
$\mathrm{H}(7)$ & $0,750(9)$ & $0,181(5)$ & $0,241(6)$ \\
$\mathrm{H}(8)$ & $0,727(10)$ & $0,058(5)$ & $0,343(6)$ \\
$\mathrm{H}(9)$ & $0,616(7)$ & $0,186(4)$ & $0,391(5)$
\end{tabular}

$B\left(\AA^{2}\right)$

$2,83(7)$

$5,0(2)$

$4,1(2)$

$3,9(2)$

$3,8(2)$

$3,5(1,1)$

$4,0(1,1)$

$4,9(1,2)$

$4,0(1,1)$

$3,5(1,1)$

$3,0(1,2)$

$4,0(1,2)$

$4,6(1,3)$

$2,1(9)$
$F_{o}$-Werte jeweils den Fehler aus der Zählstatistik ein. setzten (Stout \& Jensen, 1968).

Tabelle 2. Daten zur Anisotropie der thermischen Schwingung für die Atome von Bis(trimethylsilyl)diimin mit Ausnahme der $\mathrm{H}$-Atome

Von den Parametern $\beta_{i j}$ des Temperaturfaktors exp $\left[-\left(\beta_{11} h^{2}+\right.\right.$ $\left.\left.\beta_{22} k^{2}+\beta_{33} l^{2}+2 \beta_{12} h k+2 \beta_{13} h l+2 \beta_{23} k l\right)\right]$ sind die $10^{4}$-fachen Werte aufgeführt. Die Werte $r_{1}, r_{2}$ und $r_{3}$ beziehen sich auf die Hauptachsen des Schwingungsellipsoids; ihr Betrag entspricht jeweils der Wurzel aus dem mittleren Amplitudenquadrat. $r_{i}, a$, $r_{i}, b$ und $r_{i}, c^{*}$ sind die Winkel in Grad zwischen den Hauptachsen und den Achsen eines Orthogonalsystems, das von den Vektoren $\mathbf{a}, \mathbf{b}$ und $\mathbf{c}^{*}=(1 / V) \mathbf{a} \times \mathbf{b}$ aufgespannt wird. Standardabweichungen in Klammern wie bei Tabelle 1.

\begin{tabular}{lccccc} 
& $\mathrm{Si}$ & $\mathrm{N}$ & $\mathrm{C}(1)$ & $\mathrm{C}(2)$ & $\mathrm{C}(3)$ \\
$\beta_{11}$ & $231(5)$ & $453(15)$ & $358(16)$ & $322(15)$ & $257(13)$ \\
$\beta_{22}$ & $52(2)$ & $108(4)$ & $75(4)$ & $55(4)$ & $78(4)$ \\
$\beta_{33}$ & $86(2)$ & $104(5)$ & $116(6)$ & $144(7)$ & $121(6)$ \\
$\beta_{12}$ & $18(1)$ & $96(6)$ & $50(6)$ & $3(6)$ & $10(6)$ \\
$\beta_{13}$ & $12(2)$ & $13(7)$ & $20(8)$ & $27(8)$ & $-4(7)$ \\
$\beta_{23}$ & $-6(1)$ & $-9(4)$ & $10(4)$ & $-6(4)$ & $-10(4)$ \\
$r_{1}(\AA)$ & $0,166(3)$ & $0,197(5)$ & $0,181(6)$ & $0,179(7)$ & $0,191(6)$ \\
$r_{2}(\AA)$ & $0,176(3)$ & $0,201(5)$ & $0.218(6)$ & $0,226(6)$ & $0,210(6)$ \\
$r_{3}(\AA)$ & $0,220(2)$ & $0,333(5)$ & $0,275(6)$ & $0,254(5)$ & $0,253(6)$ \\
$r_{1}, a$ & $107(3)$ & $89(40)$ & $114(3)$ & $92(4)$ & $54(7)$ \\
$r_{1}, b$ & $23(2)$ & $81(55)$ & $40(6)$ & $7(5)$ & $83(13)$ \\
$r_{1}, c^{*}$ & $76(6)$ & $9(49)$ & $120(7)$ & $83(6)$ & $37(5)$ \\
$r_{2}, a$ & $113(2)$ & $54(2)$ & $81(5)$ & $122(9)$ & $69(11)$ \\
$r_{2}, b$ & $83(6)$ & $143(17)$ & $119(6)$ & $86(6)$ & $158(8)$ \\
$r_{2}, c^{* *}$ & $156(4)$ & $84(68)$ & $150(7)$ & $147(9)$ & $96(11)$ \\
$r_{3}, a$ & $29(2)$ & $36(2)$ & $26(3)$ & $147(9)$ & $137(5)$ \\
$r_{3}, b$ & $68(1)$ & $55(2)$ & $65(3)$ & $95(4)$ & $110(6)$ \\
$r_{3}, c^{* *}$ & $109(2)$ & $96(1)$ & $95(4)$ & $58(9)$ & $54(4)$ \\
\multicolumn{7}{c}{$109(1)$} & & & &
\end{tabular}

$r_{3}, c^{*}$
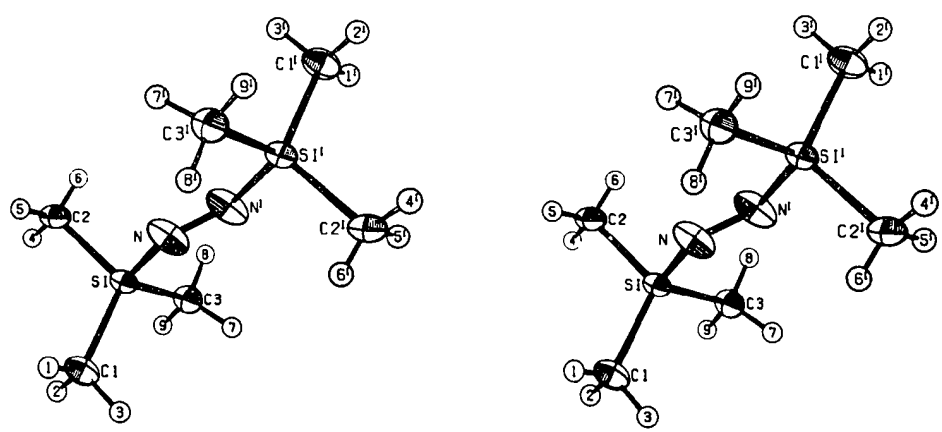

Fig. 2. Stereoskopische Darstellung eines Bis(trimethylsilyl)diimin-Moleküls. Die Blickrichtung verläuft etwa parallel zu [102].
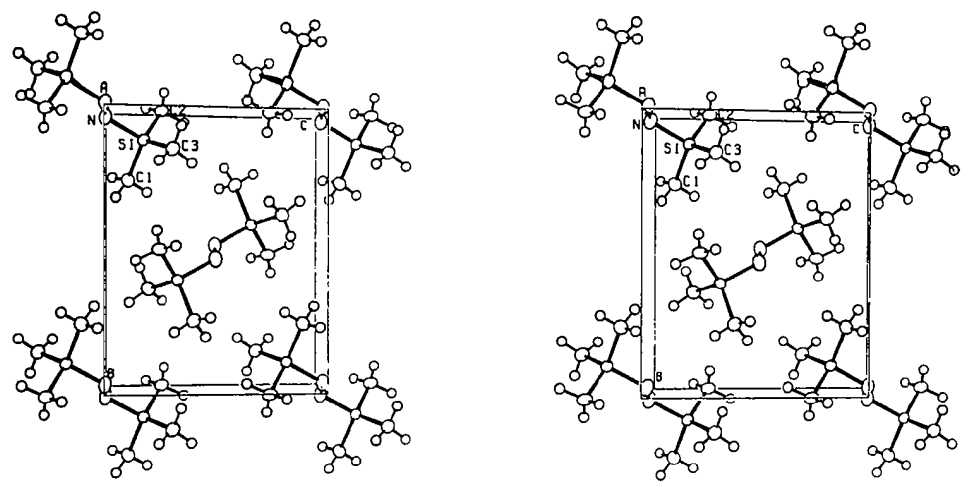

Fig. 3. Stereoskopische Darstellung einer Elementarzelle von Bis(trimethylsilyl)diimin in der Blickrichtung [i]00]. 
In den Tabellen 1 und 2. sind die Ergebnisse der Strukturbestimmung zusammengestellt; Tabelle 3 enthält den Vergleich zwischen den experimentell ermittelten Strukturfaktoren $F_{o}$ und den berechneten Werten $F_{c}$. Den letzteren liegen die Daten von Tabelle 1 und 2 zugrunde sowie die Atomformfaktoren der neutralen Atome $\mathrm{Si}, \mathrm{N}, \mathrm{C}$ und $\mathrm{H}$ in der analytischen Darstellung durch Gauss-Funktionen mit den Parametern nach Cromer \& Mann (1968).

\section{Diskussion}

Wie es den chemischen und physikalischen Eigenschaften nach erwartet werden konnte, baut sich die Kristallstruktur von Bis(trimethylsilyl)diimin aus Einzelmolekülen auf, die nur über van der Waals-Kontakte miteinander in Wechselwirkung stehen. Die Moleküle sind nach den von Kitajgorodskij (1959) für organische Moleküle aufgezeigten Prinzipien gepacкt. Eine dichte Packung ist allerdings auch hier nur hei gleichzeitiger Deformation der Einzelmoleküle erreichbar. Die Störung des Molekülfeinbaus lässt sich

Tabelle 3. Vergleich zwischen den berechneten Strukturfaktoren $F_{c}$ und den experimentell ermittelten Werten $F_{o}$ von Bis(trimethylsilyl)diimin

Wenn in der Spalte $F_{o}$ die Zahlenangabe fehlt, so hob sich der betreffende Reflex nicht signifikant vom Untergrund (Rauschpegel) ab. Die mit einem Sternchen markierten Reflexe hatten sehr hohe Zählraten und wurden nicht in die Strukturverfeinerung einbezogen.

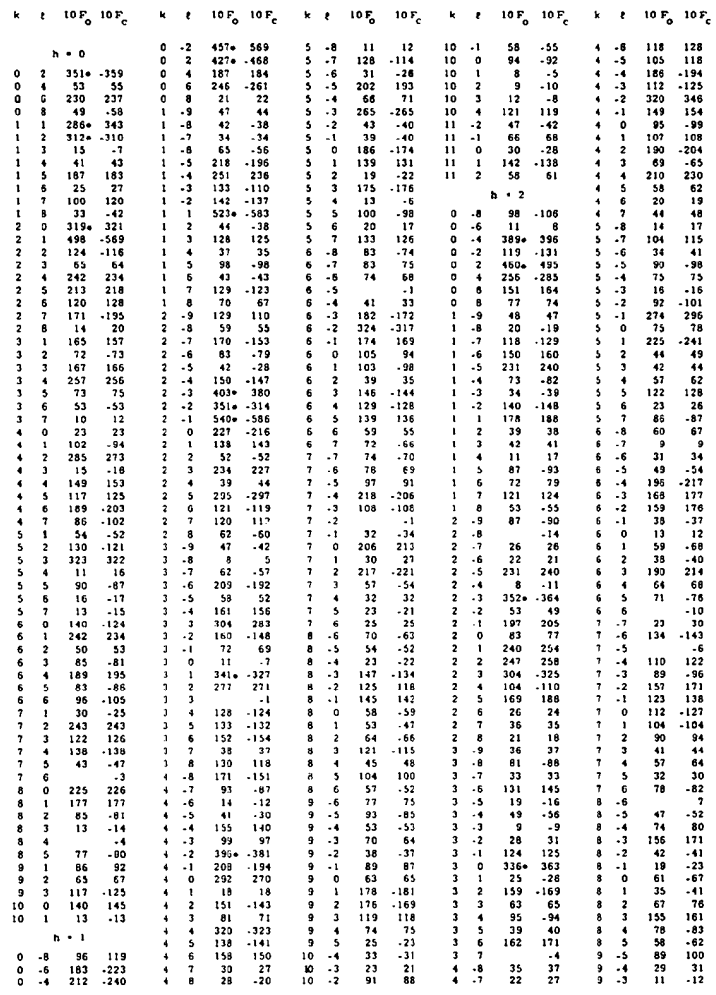

Tabelle 3 (Fort.)

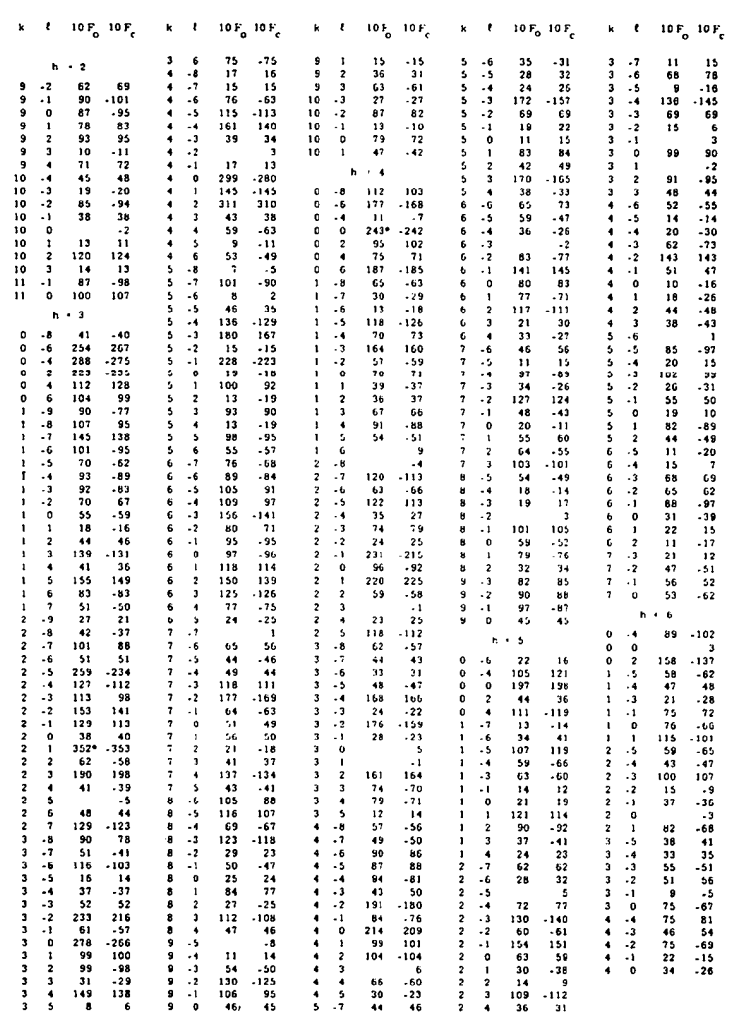

aber an Hand der besonderen Verteilung der intermolekularen Abstände gut überblicken, so dass ein recht zuverlässiges Bila über das freie Molekül aus den Gegebenheiten in der Kristallstruktur entwickelt werden kann.

Molekülsymmetrie und stereochemische Aktivität der freien Elektronenpaare

$\mathrm{Zu}$ Beginn des Kapitels 'Strukturbestimmung' war gezeigt worden, dass Bis(trimethylsilyl)diimin im Kristall die Punktsymmetrie $\bar{T}\left(C_{i}\right)$ aufweist. Das freie Molekül hat aber höchstwahrscheinlich die höhere Symmetrie $2 / m\left(C_{2 h}\right)$. Für diese Annahme sprechen im wesentlichen folgende Argumente:

(i) In der Kristallstruktur liegt zwar eine Symmetrieerniedrigung gegenüber dem idealisierten Modell vor, jedoch ist die Abweichung von der Molekülsymmetrie $2 / m$ nicht erheblich, wie man anschaulich aur den stereoskopischen Darstellungen von Fig. 2 und 3 entnehmen kann. Als Orientierungshilfe für die Betrachtung von Fig. 2 sei erwähnt, dass die Preudospiegelebene des Moleküls durch die Atome H(9), $\mathrm{C}(3), \mathrm{Si}, \mathrm{N}, \mathrm{N}^{\prime}, \mathrm{Si}^{\prime}, \mathrm{C}\left(3^{\prime}\right)$ und $\mathrm{H}\left(9^{\prime}\right)$ verläuft. Die genaue Lage dieser Ebene, die nach einem Aurgleichsverfahren zwischen den genannten Atomen ermittelt wurde (Scheringer, 1971), lässt sich durch die Gleichung $0,2154 X+0,8465 Y-0,4869 Z=0,6589$ beschreiben. $X, Y$ und $Z$ sind hierbei in $\AA$-Einheiten gc- 
messene Koordinaten des in Tabelle 2 definierten Orthogonalsystems.

(ii) Die im Kristall beobachtete Symmetrieerniedrigung des Bis(trimethylsilyl)diimin-Moleküls wird durch eine leichte Torsion verursacht, welche offensichtlich auf den störenden Einfluss von Nachbarmolekülen zurückgeführt werden kann. Eine genauere Analyse der Nachbarschaftsverhältnisse zeigt nämlich, dass ein direkter Zusammenhang zwischen dem Drehsinn der Torsion und zwei auffällig kurzen intermolekularen Abständen besteht. Fs sind dies die beiden symmetrieäquivalenten Abstände $\mathrm{N}-\mathrm{H}\left(5^{v}\right)$ und $\mathrm{N}^{\prime}-\mathrm{H}\left(5^{5^{\prime}}\right)$, die zwischen translatorisch identischen Molekülen längs [100] auftreten (vgl. Tabelle 7). Der Torsionswinkel [Diederwinkel $\mathrm{C}(3)-\mathrm{Si}-\mathrm{N}-\mathrm{N}^{\prime}$ ] beträgt $19,3^{\circ}$.

(iii) Die der Molekülsymmetrie $2 / m$ entsprechende Konformation von Bis(trimethylsilyl)diimin hat stereochemisch plausible Gründe. Man muss allerdings bei den Modellbetrachtungen ausser den nichtbindenden intramolekularen Kontakten zwischen den einzelnen Atomen auch den Raumbedarf des freien Elektronenpaars am Stickstoff berücksichtigen. Nimmt man für das Stickstoffatom eine $s p^{2}$-Hybridisierung an, die wegen des $\mathrm{Si}-\mathrm{N}-\mathrm{N}^{\prime}$-Winkels von $120^{\circ}$ naheliegt (Tabelle 4), so befindet sich das freie Elektronenpaar gemäss Fig. 2 gerade in der Lücke über $C(1)$ und $C(2)$. also in einer räumlich günstigen Position, und man versteht auch, dass damit die freie Drehbarkeit der Trimethylsilylgruppe um die $\mathrm{Si}-\mathrm{N}$-Bindung eingeschränkt ist.

In Umkehrung der eben dargelegten stereoche-

Tabelle 4. Bindungsabstände $(\AA)$ und Bindungswinkel $\left({ }^{\circ}\right)$ in Bis(trimethylsilyl)diimin

Die in Klammern angegebenen Standardabweichungen berücksichtigen ausser der Koordinatenungenauigkeit (Tabelle 1) auch den Fehlereinfluss der Gitterkonstanten.

$\begin{array}{ll}\text { N-N' } & 1,171(7) \\ \text { Si-N } & 1,808(3) \\ \text { Si-C(1) } & 1,856(4) \\ \text { Si-C(2) } & 1,850(5) \\ \text { Si-C(3) } & 1,854(6) \\ & \\ \text { C(1)-H(1) } & 0,91(5) \\ \text { C(1)-H(2) } & 0,95(6) \\ \text { C(1)-H(3) } & 1,02(6) \\ \text { C }(2)-H(4) & 1,07(6) \\ \text { C(2)-H(5) } & 0,95(5) \\ \text { C(2)-H(6) } & 0,85(5) \\ \text { C(3)-H(7) } & 1,05(6) \\ \text { C(3)-H(8) } & 0,99(6) \\ \text { C(3)-H(9) } & 0,99(5) \\ & \\ \text { Si-N-N' } & 120,0(4) \\ & \\ \text { C(1)-Si-N } & 105,0(2) \\ \text { C(2)-Si-N } & 104,6(2) \\ \text { C(3)-Si-N } & 110,9(2) \\ \text { C(1)-Si-C(2) } & 111,5(2) \\ \text { C(2)-Si-C(3) } & 112,9(2) \\ \text { C(3)-Si-C(1) } & 111,5(2)\end{array}$

$\mathrm{H}(1)-\mathrm{C}(1)-\mathrm{Si}$ $\mathrm{H}(2)-\mathrm{C}(1)-\mathrm{Si}$ $\mathrm{H}(3)-\mathrm{C}(1)-\mathrm{Si}$

$\mathrm{H}(1)-\mathrm{C}(1)-\mathrm{H}(2)$

$\mathrm{H}(2)-\mathrm{C}(1)-\mathrm{H}(3)$

$\mathrm{H}(3)-\mathrm{C}(1)-\mathrm{H}(1)$

$\mathrm{H}(4)-\mathrm{C}(2)-\mathrm{Si}$

$\mathrm{H}(5)-\mathrm{C}(2)-\mathrm{Si}$

$\mathrm{H}(6)-\mathrm{C}(2)-\mathrm{Si}$

$\mathrm{H}(4)-\mathrm{C}(2)-\mathrm{H}(5)$

$\mathrm{H}(5)-\mathrm{C}(2)-\mathrm{H}(6)$

$\mathrm{H}(6)-\mathrm{C}(2)-\mathrm{H}(4)$

$\mathrm{H}(7)-\mathrm{C}(3)-\mathrm{Si}$

$\mathrm{H}(8)-\mathrm{C}(3)-\mathrm{Si}$

$\mathrm{H}(9)-\mathrm{C}(3)-\mathrm{Si}$

$\mathbf{H}(7)-\mathrm{C}(3)-\mathbf{H}(8)$

$\mathbf{H}(8)-\mathbf{C}(3)-\mathbf{H}(9)$

$\mathbf{H}(9)-\mathrm{C}(3)-\mathbf{H}(7)$ mischen Betrachtung haben wir versucht, durch das Studium von Feinheiten der Molekülgeometrie eine Aussage über den Ladungsschwerpunkt des freien Elektronenpaars zu erhalten. Für den Abstand des Ladungsschwerpunkts vom Kern ces $\mathrm{N}$-Atoms ergab sich dabei der Wert $0,35 \pm 0,05 X$, abgetragen auf der Gegenrichtung zur Winkelhalbierenden des Si-N-N'Winkels. Zum Vergleich können Ergebnisse aus quantenmechanischen Rechnungen herangezogen werden. Nach Robh, Haines \& Csizmadia (1973) beträgt der entsprechende Abstand bei Ammoniak 0,36:8 $\AA$. und für das uns besonders interessierende Diimin $\mathrm{H}_{2} \mathrm{~N}_{2}$ fand Ahlrichs (1973) hei SCF-Rechnungen unter Verwendung einer Huzinaga-Gaussfunktionenbasis den Wert $0,388 \AA$. Wir möchten hier nicht näher auf Einzelheiten unserer Interpretation der Nachbarschaftsverhältnisse, die zu dem oben genannten Wert geführt haben, eingehen, werden aber gegen Ende der Diskussion nochmals auf Befunde hinweisen, welche die stereochemische Aktivität oes freien Elektronenpaars unmittelbar erkennen lassen.

\section{Intramolekulare Bindungsverhältnisse}

Obwohl sich Bis(trimethylsilyl)diimin in seinem Molekülbau nicht grundlcgend von anderen transAzoverbindungen unterscheidet, nimmt es doch eine gewisse Sonderstellung ein (vgl. Tabelle 5). Besonders auffällig ist die starke Verkürzung des $\mathrm{N}-\mathrm{N}$-Abstands gegenüber einer 'normalen' Stickstoffdoppelbindung von $1,24 \AA$ (Pauling, 1968). Der gefundene Wert $1,17 \AA$ entspricht gerade der N-N-Bindungslänge von ionischen Aziden (Müller, 1973). Auffällig ist ferner, dass der Winkel N-N-Si mit 120,0 deutlich über den entsprechenden Werten aller anderen Azoverbindungen von Tabelle 5 liegt. In diesem Zusammenhang muss aber betont werden, dass die beobachtete Winkelaufweitung viel geringer ist, als ursprünglich angenommen worden war (vgl. Einleitung), und so liegt es nahe, die experimentelle Basis der früheren Aussage in Zweifel zu ziehen. Der Zweifel erscheint um so mehr berechtigt, als inzwischen bei einer röntgenographischen Strukturuntersuchung von Triphenylsilylazid (Corey, Cody, Glick \& Radonovich, 1973) für den N-N-Si.Winkel in guter Übereinstimmung mit dem oben genannten Wert $120,5^{\circ}$ gefunden wurden.

Vergleicht man die für Bis(trimethylsilyl)diimin ermittelte $\mathrm{Si}-\mathrm{N}$-Bindungslänge mit neueren Daten aus der Literatur (Tabelle 6), so zeichnet sich auch hier eine Sonderstellung der Verbindung ab. Der gemessene Wert ist nämlich mit rund $1,81 \AA$ der längste bisher bekannte $\mathrm{Si}-\mathrm{N}$-Abstand überhaupt; er entspricht gemäss Tabelle 6 gerade einer $\mathrm{Si}-\mathrm{N}$-Einfachbindung. Die zum Teil erhebliche Bindungsverstärkung bei den anderen Substanzen der Tabelle wird in der Literatur häufig durch eine $d_{\pi}-p_{\pi}$ Wechselwirkung zwischen $\mathrm{Si}$ und $\mathrm{N}$ gedeutet. Im Falle von Bis(trimethylsilyl)diimin spielen solche Effekte offenbar keine Rolle, so dass die ursprünglichen Bindungsvorstellungen etwas revidiert werden müssen. 
Der auffällig langen $\mathrm{Si}-\mathrm{N}$-Bindung stehen $\mathrm{Si}-\mathrm{C}$ Abstände gegenüber, die durchweg etwas kürzer sind als erwartet. Die Abweichung vom Mittelwert gut gesicherter Literaturdaten $(1,865 \AA$, International Tables for X-ray Crystallography, 1968) beträgt allerdings bei jedem einzelnen Abstand nicht mehr als das Dreifache der zugehörigen Standardabweichung (Tabelle 4), ist also kaum noch signifikant.

Entsprechend einer allgemeinen Beobachtung bei röntgenographischen Strukturbestimmungen (Churchill, 1973) sind auch im Falle von Bis(trimethylsilyl)diimin die ermittelten $\mathrm{C}-\mathrm{H}$-Abstände der Methylgruppen (Tabelle 4) um durchschnittlich $0,1 \AA$ kürzer als der korrekte Wert 1,08 $\AA$; die $\mathrm{C}$-H-Bindungsrichtungen erleiden dagegen keine systematische Verfälschung. Es erschien uns daher angebracht, die Lageparameter der H-Atome so zu korrigieren, dass sich unter Beibehaltung der jeweils gefundenen $\mathrm{C}-\mathrm{H}$ Bindungsrichtung der zugehörige Atomabstand zu $1,08 \AA$ ergibt. Wie aus Tabelle 7 hervorgeht, ist die Auswirkung dieser Korrektur auf die nicht-bindenden intra- und intermolekularen Abstände der H-Atome teilweise recht erheblich. Sie führt aber zweifellos zu physikalisch sinnvolleren Werten, und so möchten wir den Daten in Spalte I von Tabelle 7 den Vorzug geben. Übrigens haben wir eine kritische Auswertung der
Wasserstofflagen vor allem deshalb unternommen, weil feinere Details der Molekül- und Kristallstruktur

Tabelle 7. Die kürzesten intermolekularen $\mathrm{H}-\mathrm{H}-$ Abstände in der Kristallstruktur von Bis(trimethylsilyl)diimin sowie die nicht-bindenden inter- und intramolekularen Kontakte des N-Atoms

Den Werten der Spalte I liegen berechnete Koordinaten der H-Atome zugrunde (vgl. Text), den Werten der Spalte II die experimentellen Daten von Tabelle 1. Spalte III enthält die Abstände zwischen dem Ladungsschwerpunkt des freien Elektronenpaars und den Nachbarn des N-Atoms (Koordinaten der $\mathrm{H}$-Atome wie bei Spalte I). Den hochgestellten Kennziffern für symmetrieäquivalente Atome entsprechen die Transformationen (i) $1-x,-y,-z$; (ii) $x, \frac{1}{2}-y,-\frac{1}{2}+z$; (iii) $1-x,-y, 1-z$; (iv) $1+x, y, z ;$ (v) $-x,-y,-z$.

\begin{tabular}{|c|c|c|c|c|c|c|}
\hline & I $(\AA)$ & II $(\AA)$ & & $\mathrm{I}(\AA)$ & II $(\AA)$ & $(\AA)$ \\
\hline$H(3)--H\left(4^{i 1}\right)$ & 2,38 & 2,42 & $\mathrm{~N}-\mathrm{C}(2)$ & & 2,894 & 3,01 \\
\hline$H\left(6^{i}\right)-H\left(7^{i i}\right)$ & 2,44 & 2,64 & $\mathrm{~N}-\mathrm{C}(1)$ & & 2,907 & 2,92 \\
\hline $\mathrm{H}(3)-\mathrm{H}\left(9^{\mathrm{ii}}\right)$ & 2,51 & 2,63 & $\mathrm{~N}-\mathrm{C}\left(3^{\mathrm{i}}\right)$ & & 3,169 & 2,95 \\
\hline $\mathrm{H}(8)-\mathrm{H}\left(4^{\mathrm{ii} i}\right)$ & 2,67 & 2,73 & & & & \\
\hline $\mathbf{H}(8)-\mathbf{H}\left(4^{\text {iv }}\right)$ & 2,68 & 2,73 & $\mathrm{~N}-\mathrm{H}\left(5^{\mathrm{v}}\right)$ & 2,88 & 3,00 & 2,56 \\
\hline & & & $\mathrm{N}-\mathrm{H}(6)$ & 2,93 & 2,89 & 3,09 \\
\hline $\mathrm{H}\left(2^{\mathrm{ii}}\right)-\mathrm{H}\left(5^{\mathrm{v}}\right)$ & 2,80 & 2,95 & $\mathrm{~N}-\mathrm{H}(5)$ & 3,06 & 3,02 & 3,0 \\
\hline $\mathbf{H}(1)-\mathbf{H}\left(5^{v}\right)$ & 2,80 & 2,98 & $\mathrm{~N}-\mathrm{H}\left(7^{i}\right)$ & 3,09 & 3,09 & 2,87 \\
\hline $\mathrm{H}\left(6^{\mathrm{i}}\right)-\mathrm{H}\left(3^{\mathrm{ii}}\right)$ & 2,80 & 3,00 & $\mathrm{~N}-\mathrm{H}\left(8^{i}\right)$ & 3,11 & 3,10 & 2,83 \\
\hline $\mathrm{H}(8)-\mathrm{H}\left(5^{\mathrm{iv}}\right)$ & 2,82 & 2,89 & $\mathrm{~N}-\mathrm{H}(7)$ & 3,11 & 3,11 & 3,41 \\
\hline $\mathrm{H}\left(8^{\mathrm{i}}\right)-\mathrm{H}\left(3^{\mathrm{ii}}\right)$ & 2,83 & 2,92 & $\mathrm{~N}-\mathrm{H}(1)$ & 3,14 & 3,07 & 3,03 \\
\hline $\mathrm{H}\left(9^{\mathrm{ii}}\right)-\mathrm{H}\left(1^{\mathrm{iv}}\right)$ & 2,83 & 2,98 & $\mathrm{~N}-\mathrm{H}(3)$ & 3,17 & 3,15 & 3,18 \\
\hline
\end{tabular}

\section{Tabelle 5. Vergleich von Bindungslängen und -winkeln für eine Auswahl von Azoverbindungen, in denen eine Transanordnung der Atomgruppierung $\mathrm{X}-\mathrm{N}=\mathrm{N}-\mathrm{X}$ vorliegt}

Die Zahlen in Klammern sind Standardabweichungen. Die jeweils verwendete Untersuchungsmethode ist durch folgende Kennziffern angegeben: (I) Röntgenbeugung an Einkristallen; (II) Elektronenbeugung am Gas; (III) Quantenmechanische Berechnung, SCF-Näherung.

Bis(trimethylsilyl)diimin (vorliegende Arbeit)

Diimin (Ahlrichs, 1973)

Difluordiimin (Bohn \& Bauer, 1967)

Azobenzol (Brown, 1966)

4,4'-Dichlorazobenzol (Hope \& Victor, 1969)

2,2'-Dichlorazobenzol (Komeyama, Yamamoto, Nishimura \&

Hasegawa, 1973)

1,4-Bis-( $N$-Äthyl-1,2-dihydrobenzthiazol-2-yliden)tetrazen (Allmann, 1967)

Tetrakis(trimethylsilyl)tetrazen (Veith, 1973)

Methode
I
II
I
I
I
I
I

\begin{tabular}{llll} 
& \multicolumn{2}{c}{ Abstände in $\AA$} & Winkel $\left({ }^{\circ}\right)$ \\
$\mathrm{X}$ & $\mathrm{N}-\mathrm{N}$ & $\mathrm{N}-\mathrm{X}$ & $\mathrm{N}-\mathrm{N}-\mathrm{X}$ \\
$\mathrm{Si}$ & $1,171(7)$ & $1,808(3)$ & $120,0(4)$ \\
$\mathrm{H}$ & $1,21(1)$ & $1,02(1)$ & $109(2)$ \\
$\mathrm{F}$ & $1,230(3)$ & $1,396(2)$ & $105,5(2)$ \\
$\mathrm{C}$ & $1,243(3)$ & $1,433(3)$ & $113,6(2)$ \\
$\mathrm{C}$ & $1,252(5)$ & $1,443(5)$ & $112,6(4)$ \\
$\mathrm{C}$ & $1,254(8)$ & $1,432(9)$ & $112,8(6)$ \\
$\mathrm{N}$ & $1,257(4)$ & $1,400(4)$ & $110,6(3)$ \\
$\mathrm{N}$ & $1,268(7)$ & $1,394(5)$ & $112,4(4)$
\end{tabular}

Tabelle 6. Der beobachtete Bereich für Si-N-Bindungslängen

(Vgl. die Erläuterungen von Tabelle 5).

Bis(dioxan)kalium-bis(trimethylsilyl)amid (Domingos \& Sheldrick, 1974)

Bis(trimethylsilyl)amido-bis(triphenylphosphin)nickel(I) (Bradley, Hursthouse, Smallwood \& Welch, 1972)

Bis[bis(trimethylsilyl)amido]beryllium(II) (Clark \& Haaland, 1970)

Disilazan (Rankin, Robiette, Sheldrick, Sheldrick, Aylett, Ellis \& Monaghan, 1969)

Tetrasilylhydrazin (Glidewell, Rankin, Robiette \& Sheldrick, 1970)

Tris[bis(trimethylsilyl)amido]eisen(III) (Hursthouse \& Rodesiler, 1972)

Triphenylsilylazid (Corey, Cody, Glick \& Radonovich, 1973)

Trimethylsilylisocyanat (Kimura, Katada \& Bauer, 1966)

Trimethylsilylisothiocyanat (Kimura, Katada \& Bauer, 1966)

Tetrakis(trimethylsilyl)tetrazen (Veith, 1973)

Bis(trimethylsilyl)diimin (vorliegende Arbeit)

$\mathrm{Si}-\mathrm{N}$-Einfachbindung unter Berücksichtigung der Schomaker-Stevenson-Korrektur (Pauling, 1968)

$\begin{array}{cl}\text { Methode } & \mathrm{Si}-\mathrm{N}(\AA) \\ \text { I } & 1,64(1) \\ \text { I } & 1,70(1) \\ \text { II } & 1,722(7) \\ \text { II } & 1,725(3) \\ \text { II } & 1,731(4) \\ \text { I } & 1,731(3) \\ \text { I } & 1,74(2) \\ \text { II } & 1,76(2) \\ \text { II } & 1,78(2) \\ \text { I } & 1,780(4) \\ \text { I } & 1,808(3) \\ & 1,81\end{array}$


im wesentlichen von den Wasserstoffatomen bestimmt werden, was wir im folgenden noch näher ausführen wollen.

\section{Konformations- und Packungsbetrachtungen}

Von Interesse sind zunächst die Positionen der HAtome relativ zu ihren Nachbaratomen im Molekül. Wie aus den Newman-Projektionen längs der drei kristallographisch ungleichwertigen $\mathrm{C}-\mathrm{Si}$-Bindungen hervorgeht (Fig. 4), stehen alle neun H-Atome auf Lücke. Die Abweichungen vom Idealbild sind durchweg gering; lediglich bei der Methylgruppe des Atoms C(3) zeichnet sich eine Störung ab, die schon als signifikant gelten kann. Der hier beobachtete Torsionswinkel von $7^{\circ}$ ist aber leicht erklärbar: offensichtlich werden die Atome $\mathrm{H}(7)$ und $\mathrm{H}(8)$ stärker vom Atom $\mathrm{N}^{\prime}$ als von $\mathrm{N}$ beeinflusst, wobei vor allem das freie Elektronenpaar von $\mathrm{N}^{\prime}$ wirksam ist. So gehören gemäss Tabelle 7 , Spalte III, die Abstände $\mathrm{H}(7)-\mathrm{N}^{\prime}$ und $\mathrm{H}(8)-\mathrm{N}^{\prime}$ zu den kürzesten nicht-bindenden Kontakten (man beachte, dass in der Tabelle symmetrieäquivalente Werte angegeben sind), und für den Diederwinkel $\mathrm{H}(7)-\mathrm{C}(3)$ $\mathrm{Si}-\mathrm{N}^{\prime}$ findet man $57,3^{\circ}$, also einen Wert, der erwartungsgemäss gerade um den Betrag des Torsionswinkels grösser ist als der Diederwinkel $\mathrm{H}(7)-\mathrm{C}(3)-\mathrm{Si}-$ N.

Die gegebene Interpretation, wonach das freie Elektronenpaar von $\mathrm{N}^{\prime}$ auf die Methylgruppe des Atoms $\mathrm{C}(3)$ einen erheblichen Einfluss hat, wird noch durch einen weiteren Befund gestützt. Der Bindungswinkel $\mathrm{C}(3)-\mathrm{Si}-\mathrm{N}$ ist nämlich mit rund $111^{\circ}$ um etwa $6^{\circ}$ grösser als die vom chemischen Standpunkt aus gleichwertigen Winkel $\mathrm{C}(1)-\mathrm{Si}-\mathrm{N}$ und $\mathrm{C}(2)-\mathrm{Si}-\mathrm{N}$ (vgl. Tabelle 4), d.h. die Trimethylsilylgruppe, die im ungestörten Zustand die Symmetrie $3 m\left(C_{3 v}\right)$ hat, ist in einer Weise verzerrt, aus der unmittelbar die abstossende Wirkung des freien Elektronenpaars hervorgeht.

Die Anordnung der Bis(trimethylsilyl)diimin-Moleküle in der Kristallstruktur und deren Deformation lässt sich auf der Basis des von Kitajgorodskij (1959) entwickelten geometrischen Modells der dichten Packung von Molekülen gut verstehen. Als Beleg seien folgende Gesichtspunkte genannt:

(i) Bis(trimethylsilyl)diimin kristallisiert in der Raumgruppe $P 2_{1} / c$, die nach Kitajgorodskij für eine dichte Packung organischer Moleküle optimal geeignet ist und dementsprechend am häufigsten auftritt.

(ii) Die Anordnung der Moleküle in Fig. 3 entspricht weitgehend einer Prinzipskizze von Kitajgorodskij (1959, Fig. 58). Diese zeigt, wie sich zentrosymmetrische Moleküle aneinander lagern müssen, wenn eine Schicht mit möglichst dichter Packung entstehen soll. Dass die Moleküle von Bis(trimethylsilyl)diimin tatsächlich in der abgebildeten, zur $b-c$-Ebene parallelen Schicht am dichtesten gepackt sind, wird durch die Verteilung der intermolekularen $\mathbf{H}-\mathrm{H}-\mathrm{Abstände}$ (Tabelle 7) unterstrichen: Die kleinsten Werte treten nämlich ausschliesslich zwischen den zu dieser Schicht gehörigen Molekülen auf.
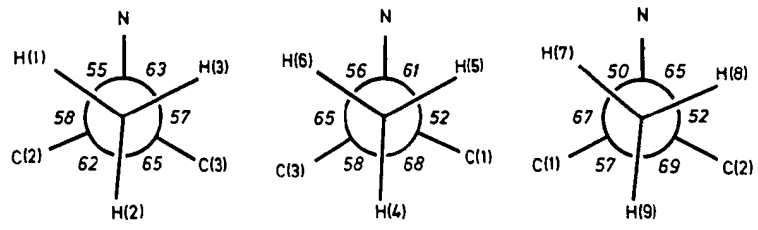

Fig. 4. Massstabgerechte Newman-Projektionen längs der $\mathrm{C}(1)-\mathrm{Si}-, \mathrm{C}(2)-\mathrm{Si}$ - und $\mathrm{C}(3)-\mathrm{Si}-\mathrm{Bindung}$ von $\mathrm{Bis}($ trimethylsilyl)diimin. Die Winkelwerte sind in Grad angegeben.

(iii) Nach Kitajgorodskij erleiden Moleküle mit einer höheren Punktsymmetrie als $\bar{I}$ beim Aufbau der Kristallstruktur fast stets eine Symmetrieerniedrigung, da in der Regel nur bei gleichzeitiger Deformation der Einzelmoleküle eine hohe Packungsdichte realisiert werden kann. Auch Bis(trimethylsilyl)diimin hat im Kristall nicht die für das freie Molekül als wahrscheinlich erkannte Symmetrie 2/m (vgl. den ersten Unterabschnitt der Diskussion); vielmehr bleibt in Übereinstimmung mit den Modellvorstellungen von Kitajgorodskij nur das Symmetriezentrum erhalten.

(iv) Die Deformation der Einzelmoleküle ist nicht mit einer Verfälschung von Bindungslängen und -winkeln verbunden; gestört wird lediglich die Konformation. Dabei spielt nach Kitajgorodskij die freie Drehbarkeit von Molekülresten um Einfachbindungen eine entscheidende Rolle. Im vorliegenden Falle ergibt sich die beobachtete Konformation durch eine Drehung der Trimethylsilylgruppe um die $\mathrm{Si}-\mathrm{N}$ Bindung.

\section{Zusammenstellung der verwendeten Rechenprogramme}

Sämtliche Berechnungen wurden an der Anlage UNIVAC 1108 des Rechenzentrums der Universität Karlsruhe $(\mathrm{TH})$ durchgeführt unter Verwendung der folgenden Programme: In ALGOL übersetzte und modifizierte Datenverarbeitungsprogramme zum Zweikreisdiffraktometer der Firma Stoe, Darmstadt; Programm zur Berechnung von Fourier- und Patterson-Synthesen (Zweerus, 1967); ORFLS (Busing, Martin \& Levy, 1962); ORFFE (Busing, Martin \& Levy, 1964); ORTEP (Johnson, 1965); Programm zur Berechnung der 'besten' Ebene durch einen Satz gegebener Atome (Scheringer, 1971).

Die Deutsche Forschungsgemeinschaft und der Fonds der Chemischen Industrie unterstützten die vorliegende Arbeit durch Sachbeihilfen.

\section{Literatur}

AhLRICHS, R. (1973). Private Mitteilung. Institut für Physikalische Chemie und Elektrochemie der Universität Karlsruhe (TH).

Allmann, R. (1967). Acta Cryst. 22, 246-251.

Bohn, R. K. \& Bauer, S. H. (1967). Inorg. Chem. 6, 309312. 
Bradley, D. C., Hursthouse, M. B., Smallwood, R. J. \& Welch, A. J. (1972). Chem. Commun. S. 872-873.

Brown, C. J. (1966). Acta Cryst. 21, 146-152.

Buerger, M. J. (1967). Vector Space, 2. Aufl., S. 239-247. New York: John Wiley.

Busing, W. R., Martin, K. O. \& Levy, H. A. (1962). ORFLS. Report ORNL-TM-305, Oak Ridge National Laboratory, Oak Ridge, Tennessee.

Busing, W. R., Martin, K. O. \& Levy, H. A. (1964). ORFFE. Report ORNL-TM-306, Oak Ridge National Laboratory, Oak Ridge, Tennessee.

Churchill, M. R. (1973). Inorg. Chem. 12, 1213-1214.

Clark, A. H. \& HaAland, A. (1970). Acta Chem. Scand. 24, 3024-3030.

Corey, E. R., Cody, V., Glick, M. D. \& Radonovich, L. J. (1973). J. Inorg. Nucl. Chem. 35, 1714-1717.

Cromer, D. T. \& ManN, J. B. (1968). Acta Cryst. A 24, 321-324.

Domingos, A. M. \& Sheldrick, G. M. (1974). Acta Cryst. B 30, 517-519.

Glidewell, C., Rankin, D. W. H., Robiette, A. G. \& SHELDRICK, G. M. (1970). J. Chem. Soc. Dalton, S. 318-325.

Hamilton, W. C. (1959). Acta Cryst. 12, 609-610.

HOPE, H. \& VICTOR, D. (1969). Acta Cryst. B 25, 1849-1853.

Hursthouse, M. B. \& Rodesiler, P. F. (1972). J. Chem. Soc. Dalton, S. 2100-2102.

International Tables for X-ray Crystallography (1968). Bd. III, 2. Aufl., S. 275. Birmingham: Kynoch Press.
JoHnson, C. K. (1965). ORTEP. Report ORNL-3794, Oak Ridge National Laboratory, Oak Ridge, Tennessee.

Kimura, K., Katada, K. \& Bauer, S. H. (1966). J. Amer. Chem. Soc. 88, 416-420.

KitajgorodskiJ, A. J. (1959). Organic Chemical Crystallography, S. 84-105. New York: Consultants Bureau.

Komeyama, M., Yamamoto, S., Nishimura, N. \& HaseGaWa, S. (1973). Bull. Chem. Soc. Japan, 46, 2606-2607.

Müller, U. (1973). Struct. Bond. 14, 141-172.

Pauling, L. (1968). Die Natur der chemischen Bindung, 3. Aufl., S. 220-222. Weinheim/Bergstr.: Verlag Chemie $\mathrm{GmbH}$.

Rankin, D. W. H., Robiette, A. G., Sheldrick, G. M., Sheldrick, W. S., Aylett, B. J., Ellis, J. A. \& MonaGHaN, J. J. (1969). J. Chem. Soc. Dalton, S. 1224-1227.

Robb, M. A., Haines, W. J. \& Csizmadia, I. G. (1973). J. Amer. Chem. Soc. 95, 42-48.

Scheringer, C. (1971). Acta Cryst. B27, 1470-1472.

Stout, H. G. \& Jensen, L. H. (1968). X-ray Structure Determination, S. 456-457. New York: Macmillan.

VEITH, M. (1973). Unveröffentlichte Ergebnisse. Universität Karlsruhe (TH).

Wiberg, N. (1971). Angew. Chem. 83, 379-392.

Wiberg, N., Joo, W. C. \& Uhlenbrock, W. (1968). Angew. Chem. 80, 661-662.

Zweerus, H. P. (1967). Programm zur Berechnung von Fourier- und Patterson-Synthesen. Laboratorium voor Kristalchemie der Rijksuniv., Utrecht, Niederlande.

Acta Cryst. (1974). B30, 1813

\title{
Structure Cristalline de l'Acide Tétraoxotellurique $\mathrm{H}_{2} \mathrm{TeO}_{4}$
}

\author{
Par Jacques Moret, Etienne Philippot et Maurice Maurin \\ Laboratoire de Chimie Minérale C, E.R.A. 314, Université des Sciences et Techniques du Languedoc, \\ Place Eugène Bataillon, 34060 Montpellier Cedex, France
}

ET Oliver Lindevist

Department of Inorganic Chemistry, Chalmers Institute of Technology and the University of Göteborg, P.O. Box, S-402 20 Göteborg 5, Suède

(Reçu le 21 janvier 1974, accepté le 8 mars 1974)

\begin{abstract}
Single crystals of $\mathrm{H}_{2} \mathrm{TeO}_{4}$ have been prepared by hydrothermal synthesis and the crystal structure determined from three-dimensional X-ray data. The crystals are monoclinic, space group $P 2_{1} / c$, with $a=5.884$ (2), $b=4.844$ (1), $c=5.224$ (1) $\AA, \beta=116.98$ (2) ${ }^{\circ}$ and $Z=2$. The structure was solved from three-dimensional Patterson and electron-density calculations and the structure parameters, excluding those of the hydrogen atoms, were refined to an $R$ value of 0.042 with 441 independent reflexions. The structure contains $\mathrm{Te}(\mathrm{VI}) \mathrm{O}_{6}$ octahedra with $\mathrm{Te}(\mathrm{VI})-\mathrm{O}$ bond distances in the range 1.903-1.930 $\AA$. The $\mathrm{Te}(\mathrm{VI}) \mathrm{O}_{6}$ octahedra are connected through four corners to form infinite sheets of composition $\left[\mathrm{Te}(\mathrm{VI}) \mathrm{O}_{2}(\mathrm{OH})_{2}\right]_{n}$. These sheets are held together by hydrogen bonds, resulting in cleavage planes in the crystals. The $\mathrm{H}_{2} \mathrm{TeO}_{4}$ structure is closely related to those of $\mathrm{Te}(\mathrm{OH})_{6}, \mathrm{Te}_{2} \mathrm{O}_{5}, \mathrm{TeO}_{3}$.
\end{abstract}

\section{Introduction}

$\mathrm{Si}$ nos connaissances structurales des oxoacides et oxydes du tellure ainsi que celles des tellurites(IV) et tellurates(VI) correspondants ont beaucoup progressé durant ces dernières années (Lindqvist, 1973; Moret, 1972), on ne connaît cependant rien de précis sur la structure de l'acide métatellurique $\mathrm{H}_{2} \mathrm{TeO}_{4}$.

Cet acide, que l'on doit considérer comme un produit de condensation de l'acide orthotellurique 